"This is the peer reviewed version of the following article: ["Mid-wave Infrared PolarizationIndependent Graphene Photoconductor with Integrated Plasmonic Nanoantennas Operating at Room Temperature" by Ming Ye, Yang Gao, Jasper J. Cadusch, Sivacarendran Balendhran, and Kenneth B. Crozier], which has been published in final form at [https://doi.org/10.1002/adom.202001854]. This article may be used for non-commercial purposes in accordance with Wiley Terms and Conditions for Use of Self-Archived Versions. This article may not be enhanced, enriched or otherwise transformed into a derivative work, without express permission from Wiley or by statutory rights under applicable legislation. Copyright notices must not be removed, obscured or modified. The article must be linked to Wiley's version of record on Wiley Online Library and any embedding, framing or otherwise making available the article or pages thereof by third parties from platforms, services and websites other than Wiley Online Library must be prohibited." 


\title{
Mid-wave Infrared Polarization-Independent Graphene Photoconductor with Integrated Plasmonic Nanoantennas Operating at Room Temperature
}

Ming Ye, Yang Gao, Jasper J. Cadusch, Sivacarendran Balendhran, and Kenneth B. Crozier*

M. Ye, Dr. Y. Gao, Dr. J. J. Cadusch, Prof. K. B. Crozier

Department of Electrical and Electronic Engineering

University of Melbourne

Parkville, VIC 3010, Australia

Dr S. Balendhran, Prof. K. B. Crozier

School of Physics

University of Melbourne

Parkville, VIC 3010, Australia

Prof. K. B. Crozier

Australian Research Council (ARC) Centre of Excellence for Transformative Meta-Optical Systems University of Melbourne

Parkville, VIC 3010, Australia

E-mail: kcrozier@unimelb.edu.au

\begin{abstract}
Graphene photodetectors operating in the mid-wave infrared (MWIR) face challenges that include the optical absorption of monolayer graphene being intrinsically low and the carrier lifetime in graphene being very short. Previous reports of graphene photoconductors in the MWIR have sought to overcome these challenges using approaches that include the integration of plasmonic nanoantennas and/or engineered electrodes. However, this has led to the photoresponse of these detectors being strongly polarization dependent. Here, a graphene photoconductor is reported that achieves polarization-independent and fast response simultaneously via the integration of plasmonic nanoantennas that we term Jerusalem-cross antennas (JC-antennas). Compared to previous works, the JC-antennas concentrate the incident light onto graphene more efficiently with enhanced polarization-independent optical absorption. MWIR detection is demonstrated at both room temperature and cryogenic temperatures, with measured responsivity of $14.5 \mathrm{VW}^{-1}$ (room temperature) and $4400 \mathrm{VW}^{-1}(78 \mathrm{~K})$. Due to the carrier collection by the JC-antennas and gapless band structure of graphene, the detector also shows significant and broadband photoresponse that extends to visible and near-infrared wavelengths. The detector shows fast temporal response with a measured rise time of $3 \mathrm{~ns}$, which would be more than sufficient for many practical applications (e.g. imaging).
\end{abstract}


KEYWORDS: Graphene, photodetectors, polarization-independent, mid-wave infrared, plasmonic nanoantenna

\section{Introduction}

Numerous applications rely on the detection of infrared (IR) light. Of particular importance are the two portions of the mid-infrared (MIR, wavelength: $\sim 2.5-25 \mu \mathrm{m}$ ) spectrum that are transmitted through the atmosphere. These have become known as the mid-wave infrared (MWIR, wavelength: 3-5 $\mu \mathrm{m}$ ) and the long-wave infrared (LWIR, wavelength: 8-12 $\mu \mathrm{m}) \cdot{ }^{[1]}$ Important applications of MIR detection include biosensing, ${ }^{[2]}$ security, ${ }^{[3]}$ spectroscopy ${ }^{[4]}$ and thermal imaging. ${ }^{[5]}$ Applications in the more specific MWIR wavelength range include gas detection and identification by spectroscopy and the imaging/tracking of high temperature objects (e.g. for aerospace). Commercially available MIR photodetectors generally make use of narrow-bandgap semiconductors such as $\mathrm{HgCdTe}(\mathrm{MCT}){ }^{[6]}$ indium antimonide (InSb), ${ }^{[7]}$ and lead selenide $(\mathrm{PbSe}),{ }^{[8]}$ and other technologies such as quantum wells $^{[9]}$ and type-II superlattices. ${ }^{[10]}$ While they achieve high performance, these devices are often expensive due to complex growth/fabrication processes. In addition, they often require cryogenic cooling, which adds considerable bulk to the system. The use of colloidal quantum dots (CQDs) to realize photodetectors has been the topic of some interest in recent years, due to the high absorption efficiency and ease of fabrication of CQDs. ${ }^{[11]}$ Thus far, only a few works ${ }^{[5 c, 12]}$ have reported MIR photodetection based on CQDs. This is because the types of CQDs that are mature from a manufacturing standpoint are unsuitable for MWIR detection, as their bandgaps are too large. While bolometers have been employed for room temperature infrared detection, their application is ultimately limited by their slow response. ${ }^{[13]}$ Lastly, it is also the case that many of the semiconductor materials currently used for MIR detection are toxic and hazardous to the environment.

Due to aforementioned considerations, there has recently been much interest concerning the development of new materials for high-performance, low-cost, and environmentally-friendly MIR photodetectors. Two-dimensional (2D) materials provide a promising platform to achieve these goals for several reasons. First, 2D materials represent a drastic reduction in the volume of the active (photosensitive) material. A major challenge for achieving room temperature detection of MIR light 
lies in the large detector noise associated with the thermal generation of charge carriers in smallbandgap semiconductor materials. ${ }^{[14]}$ One way to mitigate the thermal generation is to reduce the volume of the active material. ${ }^{[14]}$ Second, a few 2D materials $\left(\right.$ i.e. graphene ${ }^{[4 b, 15]}, \mathrm{PtSe}_{2}{ }^{[16]}, \mathrm{PdSe}_{2}{ }^{[17]}$, black phosphorus ${ }^{[18]}$, etc.) have already shown great potential in MIR detection. Some of these materials, such as graphene and $\mathrm{PtSe}_{2}$, have been reported to show MIR response only when they are in 2D-forms (e.g. monolayer or bilayer) because of their unique band structures. Third, some of these (MIR-sensitive) 2D materials, including graphene, are non-toxic and environmentally-friendly. A challenge faced by these photodetectors is that, thus far, the dominant method for adding the 2D material to the device is via mechanical exfoliation. The growth of uniform films of most 2D materials over large areas is still very challenging. However, wafer-scale graphene has been successfully grown by using chemical vapor deposition $(\mathrm{CVD})^{[19]}$, and shows excellent crystal quality and physical properties that are comparable to mechanically-exfoliated graphene. More importantly, graphene is an attractive material for developing broadband (including the MIR region) and high-speed photodetectors because of its gapless band structure and ultrafast carrier dynamics. ${ }^{[15 b, 20]}$ Graphene photodetectors have been successfully demonstrated in a broad spectral range from the visible to the terahertz wavelength regimes, ${ }^{[21]}$ and with bandwidths of $\sim 40 \mathrm{GHz}$ for optical communications. ${ }^{[21 \mathrm{~b} \text {, }}$ ${ }^{22]}$ However, due to the intrinsically-low optical absorption of graphene (less than $2.3 \%$ in the MIR) ${ }^{[23]}$ and the short (sub-picosecond) lifetime of photogenerated carriers, graphene detectors generally suffer from low responsivity. ${ }^{[24]}$ This hinders the use of graphene detectors in the MIR due to the low signal-to-noise ratio that results, especially at room temperature. A few strategies have been devised and employed to improve the responsivity of graphene detectors operating in the MIR regime. Band structure engineering and the introduction of trapping centers in graphene are direct ways to enhance the optical absorption and photoconductive gain. By leveraging such stategies, a graphene detector with a responsivity of $0.4 \mathrm{AW}^{-1}$ was demonstrated based on a graphene quantum dot (GQD) array. ${ }^{[25]}$ However, the response time in that work was limited by the long lifetime of the trapped electrons ( $\sim$ tens of seconds), and the detector operation was restricted to low temperature $(\sim 10 \mathrm{~K})$ because the 
responsivity reduced as a result of shortened carrier lifetime at higher temperatures. Photogating effects have also been used to achieve enhanced responsivity $\left(>1 \mathrm{AW}^{-1}\right)$ at room temperature by coupling graphene with narrow-bandgap semiconductor nanoparticles, ${ }^{[26]}$ or by introducing a tunneling barrier in a graphene double-layer heterostructure. ${ }^{[27]}$ Unfortunately, the charge trap-states in either the graphene-nanoparticle interface ${ }^{[26]}$ or the tunneling barrier ${ }^{[27]}$ cause the detector response time to be in the millisecond range. In a recent work, ${ }^{[28]}$ an asymmetric plasmon-induced hot-carrier Seebeck photodetector was demonstrated with a responsivity of $2900 \mathrm{VW}^{-1}$ in the LWIR region. Its response to a temporally-modulated optical signal was not measured. Instead, its response to a temporally-modulated electrical bias signal (under constant LWIR illumination) was measured and a response time of $\sim 100 \mathrm{~ns}$ was found. Plasmonic nanoantennas ${ }^{[15 \mathrm{a}]}$ and engineered electrodes ${ }^{[29]}$ have been demonstrated to improve the responsivities of graphene detectors while maintaining high operating speed by simultaneously enhancing the optical absorption and carrier collection efficiency in graphene. However, while this approach is promising, there is still much room for improvement. For instance, the detector response in these previous works was strongly polarization-dependent, due to the low symmetry of the employed nanoantennas and electrodes. In addition, the enhancement of the optical absorption was relatively modest, with the peak value of the absorption being only $\sim 10 \%{ }^{[15 \mathrm{a}]}$

Here, we demonstrate a polarization-independent graphene photoconductor with integrated Jerusalem-cross nanoantennas (JC-antennas). Our device shows significant MWIR photoresponse at room temperature, with further enhancement at cryogenic temperatures. Unlike previous methods that relied on charge trapping centers or photogating effects, our approach, in which JC-antennas are used to increase optical absorption and improve photocarrier collection, does not hinder the detector speed. Compared to the low symmetry end-to-end rod antennas (two-fold symmetry) that were employed in a previously reported graphene detector, ${ }^{[15 a]}$ our JC-antennas, with their elongated ends and narrower gaps $(\sim 40 \mathrm{~nm})$ between adjacent antennas, render stronger optical absorption enhancement in the graphene. Moreover, this absorption is polarization-independent due to the highly 
symmetric shape (four-fold symmetry) of the JC-antennas. ${ }^{[30]}$ Our device also shows strong photoresponse at visible and near-infrared wavelengths. Our device thus has a broadband photoresponse that extends from the visible to the MWIR.

\section{Results and discussions}

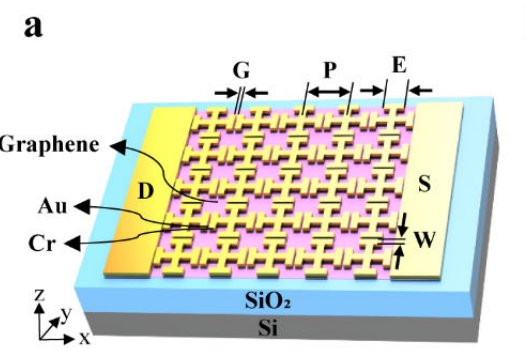

b

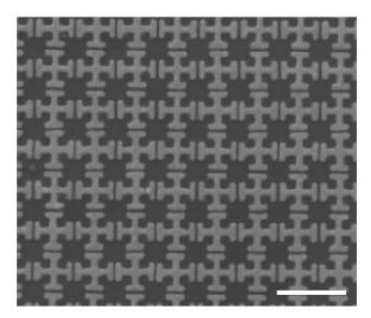

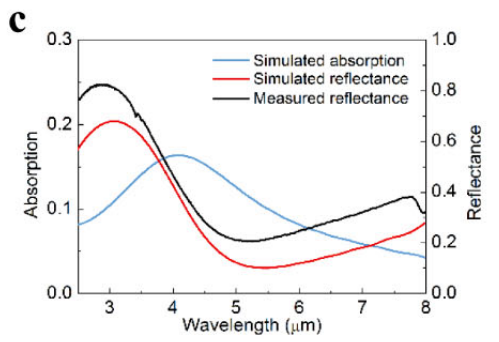

c
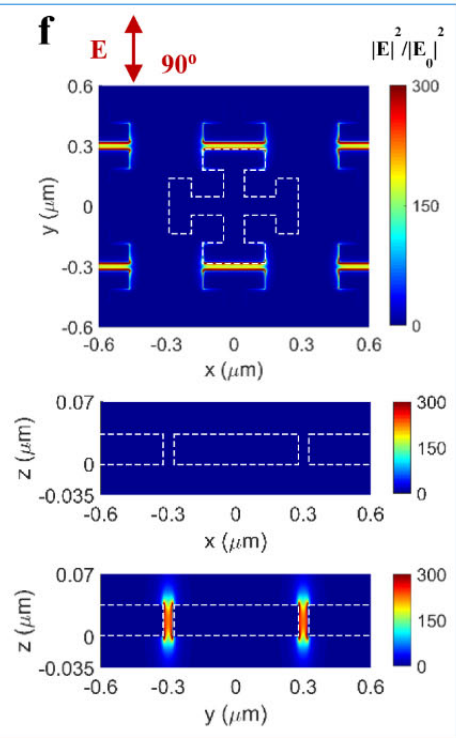

Figure 1. Design, characterization, and optical simulation of graphene photodetectors. a Schematic of graphene photodetector with integrated JC antennas. Graphene is on $\mathrm{SiO}_{2}$ layer ( $285 \mathrm{~nm}$ thick) on highly doped Si substrate. b Scanning electron microscopy (SEM) image of fabricated graphene detector (Dev\#1) with design values of $\mathrm{P}=600 \mathrm{~nm}$, width $\mathrm{W}=90 \mathrm{~nm}$, length $\mathrm{E}=270 \mathrm{~nm}$, gap $\mathrm{G}=$ $40 \mathrm{~nm}$. Scale bar is $1 \mu \mathrm{m}$. c Simulated and measured device reflectance spectra and simulated graphene absorption spectrum for device described in b. (d-f) Normalized electric field intensity distributions for incident polarization of $0^{\circ}(\mathbf{d}), 45^{\circ}$ (e) and $90^{\circ}(\mathbf{f})$, with respect to $x$-axis. Graphene is located at $\mathrm{z}=0 \mu \mathrm{m}$. Top panel: field in graphene plane. Middle \& bottom panels: field in xz- \& yzplane, respectively, through middle of antenna. Fields are obtained at wavelength of peak absorption $(\lambda=4.12 \mu \mathrm{m})$ for structure with same parameters as that of (b). White dashed lines show contour of JC-antennas.

The architecture of our graphene photoconductor with integrated JC-antennas is schematically depicted in Figure 1a. The photoconductor consists of a graphene monolayer on a 285-nm thick silicon dioxide layer thermally grown on a highly doped silicon substrate (p-type, $<0.005 \Omega \cdot \mathrm{cm}$ ). The 
graphene layer serves as both the charge transport channel and the light absorber. A square array of JC-antennas is formed on top of the graphene channel. The working principle of the photodetector is as follows. Illumination of the device results in excitation of the plasmon resonance of the antennas, leading to enhanced fields in the narrow gaps $(\sim 40 \mathrm{~nm})$ between the two long edges $(\sim 270 \mathrm{~nm})$ of adjacent antennas. This results in light absorption by the graphene and excitation of electron-hole pairs, which are collected as photocurrent (with photoconductive gain) by the antennas/electrodes under the external voltage bias. We believe that thermal effects such as photothermoelectric (PTE) effect or bolometric effect play negligible roles in contributing to the photocurrent generation in our device. The PTE effect leads to photocurrent when the electronic environment in the channel is asymmetric, which is not the case for our device. We also expect that the bolometric effect contributes very little to photocurrent generation in our device as the temperature coefficient of resistance (TCR) of single layer graphene is very small, i.e. below $0.147 \% \mathrm{~K}^{-1}$ at room temperature. ${ }^{[31]}$

We next describe the device fabrication process. A monolayer CVD-grown graphene film (purchased from Graphenea, Monolayer Graphene on $\mathrm{Cu}$ (4 Inches, $\mathrm{G} / \mathrm{Cu}-100)$ ) is transferred onto a silicon dioxide layer using a polymethyl methacrylate (PMMA)-assisted wet transfer method. A square array of 5-nm Cr/30-nm Au JC-antennas is then fabricated on top of the graphene by electron beam lithography (EBL), electron-beam evaporation and lift-off. Next, graphene is patterned to form the channels $(30 \mu \mathrm{m} \times 30 \mu \mathrm{m})$ by EBL, followed by reactive ion etching $\left(\mathrm{O}_{2}\right.$ plasma treatment for $30 \mathrm{~s}$ at $100 \mathrm{~W})$. Finally, the electrodes and wire-bonding pads are patterned by EBL and formed by electron beam evaporation of $10-\mathrm{nm} \mathrm{Cr} / 80-\mathrm{nm} \mathrm{Au}$ and lift-off. Figure $1 \mathrm{~b}$ shows a scanning electron microscope (SEM) image of a fabricated device (Dev\#1), showing JC-antennas with well-defined shapes and $\sim 40 \mathrm{~nm}$ gaps. The measured geometric parameters of the antennas are $\mathrm{P}=606 \mathrm{~nm}, \mathrm{~W}=$ $88 \mathrm{~nm}, \mathrm{E}=273 \mathrm{~nm}, \mathrm{G}=44 \mathrm{~nm}$. The reflectance spectrum (Figure 1c, black curve) is measured by a Fourier transform infrared (FTIR) spectrometer coupled to a microscope and shows reasonable agreement with the simulated (finite difference time domain, FDTD) reflectance spectrum (Figure 1c, red curve). The simulated graphene absorption spectrum (Figure 1c, light blue curve) shows a peak 
absorption of $16.4 \%$ in the MWIR at $\lambda=4.12 \mu \mathrm{m}$, which is $64 \%$ and $167 \%$ larger than that occurring in the rod-antenna configuration of a previous study $^{[15 \mathrm{a}]}$ for polarized and unpolarized light, respectively (See Note S10, Supporting Information). The strong graphene absorption arises from the enhancement of the electric field intensity by the antennas (Figure 1d-f). Due to the highly symmetric shape of our JC-antenna, strong field intensity can be generated for any incident polarization. Figure 1d-f show the normalized electric field intensity distributions for incident polarizations of $0^{\circ}$ (Figure 1d), $45^{\circ}$ (Figure 1e), and $90^{\circ}$ (Figure 1f) with respect to the x-axis. It can be seen that as the polarization changes from $0^{\circ}$ through $45^{\circ}$ to $90^{\circ}$, the region for strong field enhancement changes from the gaps along the $\mathrm{y}$-axis to the gaps along the $\mathrm{x}$-axis, maintaining strong light-graphene interaction and thus total absorption (See Figure S5 for simulated graphene absorption for different polarizations). Note that a maximum field intensity enhancement factor of $\sim 2 \times 10^{3}$ is achieved in Figure 1d,f.
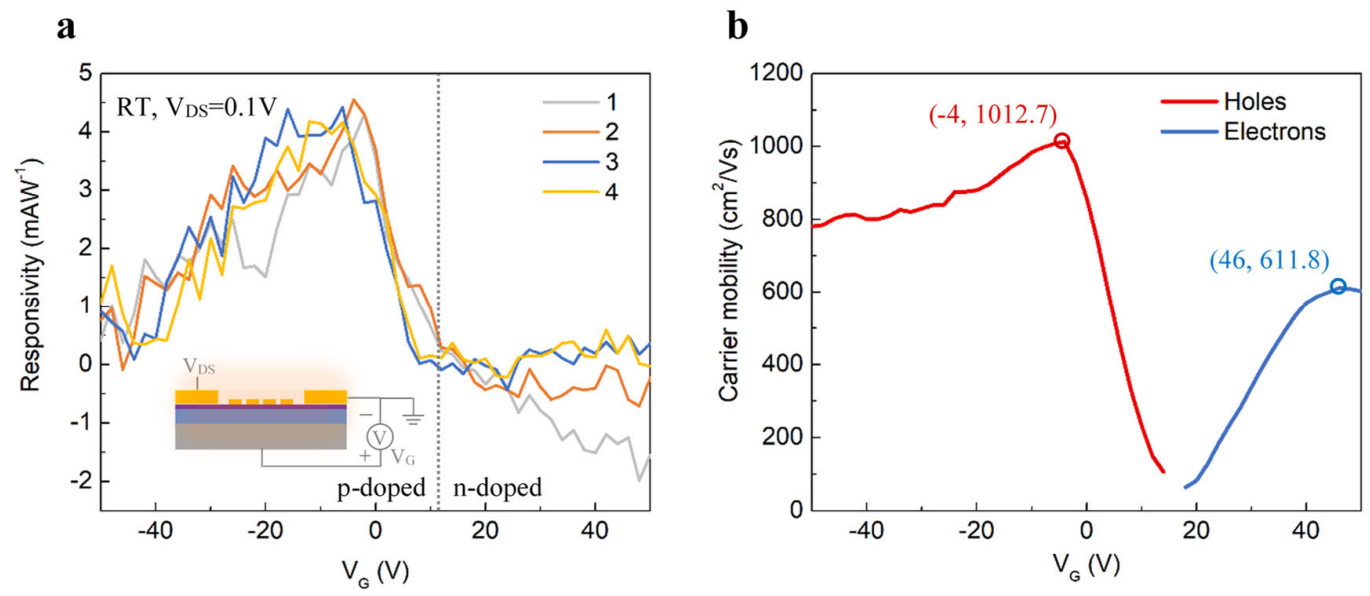

Figure 2. Device characterization of graphene photodetector with integrated JC antennas (Dev\#1) at room temperature (RT). a Measured responsivity of detector as a function of gate voltage $\left(\mathrm{V}_{\mathrm{G}}\right)$ under MWIR illumination from a globar source (illuminating power $64.6 \mu \mathrm{W}$ ). Source-drain bias voltage $V_{D S}=0.1 \mathrm{~V}$. Curves correspond to consecutive measurements. Inset: schematic of detector under test. b Extracted electron (light blue) and hole (dark red) mobilities as a function of gate voltage based on measured electrical properties.

We then characterize the device photoresponse at room temperature under MWIR illumination from a globar source. Detailed information on the characterization method is provided as Note S2, Note S3 and Note S7 (Supporting Information). Figure 2a shows the measured gate-dependent responsivity based on the measured photocurrent and the power incident onto the detector. The 
photocurrent is obtained by measuring the drain current at each gate voltage $\left(\mathrm{I}_{\mathrm{D}}-\mathrm{V}_{\mathrm{G}}\right)$ with and without IR illumination, and then taking the difference. The source-drain bias voltage is kept at $\mathrm{V}_{\mathrm{DS}}=0.1 \mathrm{~V}$. Four responsivity vs gate voltage curves are measured consecutively in this way and show consistent photoresponse (Figure 2a). The detector shows a maximum responsivity of $4.6 \mathrm{mAW}^{-1}$ for $\mathrm{V}_{\mathrm{G}} \approx-4 \mathrm{~V}$ at room temperature, which is around 2.6 times higher than the responsivity $\left(\sim 1.8 \mathrm{mAW}^{-1}\right)$ reported in previous work that employed rod antennas ${ }^{[15 \mathrm{a}]}$ at the same source-drain bias voltage. The current responsivity corresponds to a voltage responsivity of $14.5 \mathrm{VW}^{-1}$ based on the relation between the photovoltage $\left(V_{p}\right)$ and photocurrent $\left(I_{p}\right) V_{p}=I_{p} \times R_{G}$, where $R_{G}$ is the graphene channel resistance. ${ }^{[28]}$ It should be noted that the graphene is p-type doped after the transfer/fabrication processes due to the remaining of charge impurities and the metallic doping effect. ${ }^{[32]}$ Unlike previous works on graphene photoconductors, ${ }^{[33]}$ a distinct "gate-switchable" characteristic of the photoresponse is observed in our case, where the detector shows clear photoresponse ("working state" of the detector) when the graphene channel is p-type doped $\left(\mathrm{V}_{\mathrm{G}}<\mathrm{V}_{\text {Dirac }}=16 \mathrm{~V}\right)$, but negligible photoresponse ("silent state" of the detector) when graphene channel is n-type doped $\left(V_{G}>V_{\text {Dirac }}=16 \mathrm{~V}\right)$. We attribute the strong suppression of the photoresponse in the n-type doped regime of graphene to the existence of p-type impurities such as absorbed hydrocarbon molecules or from humidity, which could significantly increase the electron recombination rate. ${ }^{[26]}$ To further understand the gate-dependent photoresponse, we extract the gate-dependent carrier mobility from the measured gate-dependent electrical conductivity as shown in Figure 2b. Details on the extraction method are provided in Note S8, Supporting Information. It can be seen that as the gate voltage decreases in the p-type doped regime of graphene, the hole mobility (Figure 2b, red curve) first increases to a maximum value of 1013 $\mathrm{cm}^{2} / \mathrm{Vs}$ at $\mathrm{V}_{\mathrm{G}}=-4 \mathrm{~V}$, then decreases to $781 \mathrm{~cm}^{2} / \mathrm{Vs}$ at $\mathrm{V}_{\mathrm{G}}=-50 \mathrm{~V}$, showing a similar trend to the gatedependent photoresponse (Figure 2a). This is understandable because higher mobility permits shorter carrier transit time, which would consequently result in greater photoresponse. The initial increase and subsequent decrease of the hole mobility could be attributed to it being limited by substrate surface polar phonon scattering and short-range scattering by defects, respectively, a trend that has 
been reported previously using Hall measurements. ${ }^{[34]}$ It is notable that hole mobility and responsivity take their maximum values at a similar gate voltage $(\sim-4 \mathrm{~V})$. In addition, it can be seen that the electrons show smaller mobility (maximum $612 \mathrm{~cm}^{2} / \mathrm{Vs}$, blue curve in Figure $2 \mathrm{~b}$ ) than holes in the part of the p-type doped regime of graphene that shows significant photoresponse, which could also contribute to the suppression of the detector response in the n-type doped regime.

a

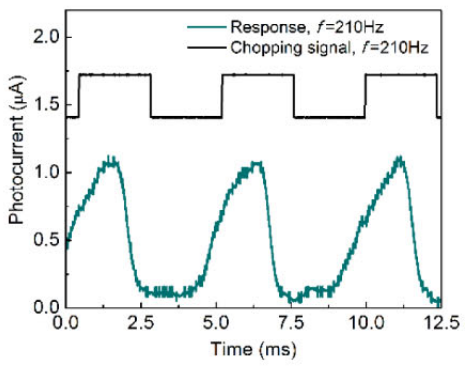

b

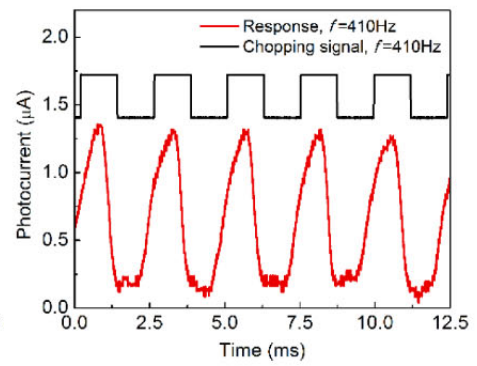

c

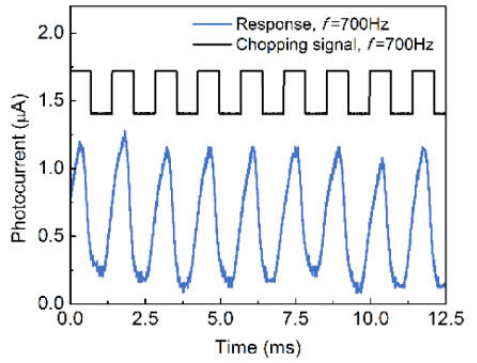

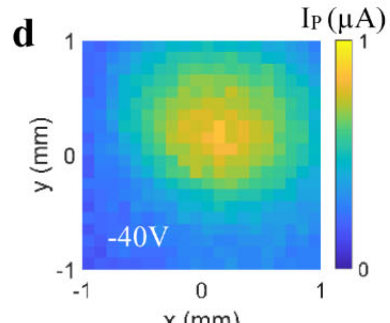
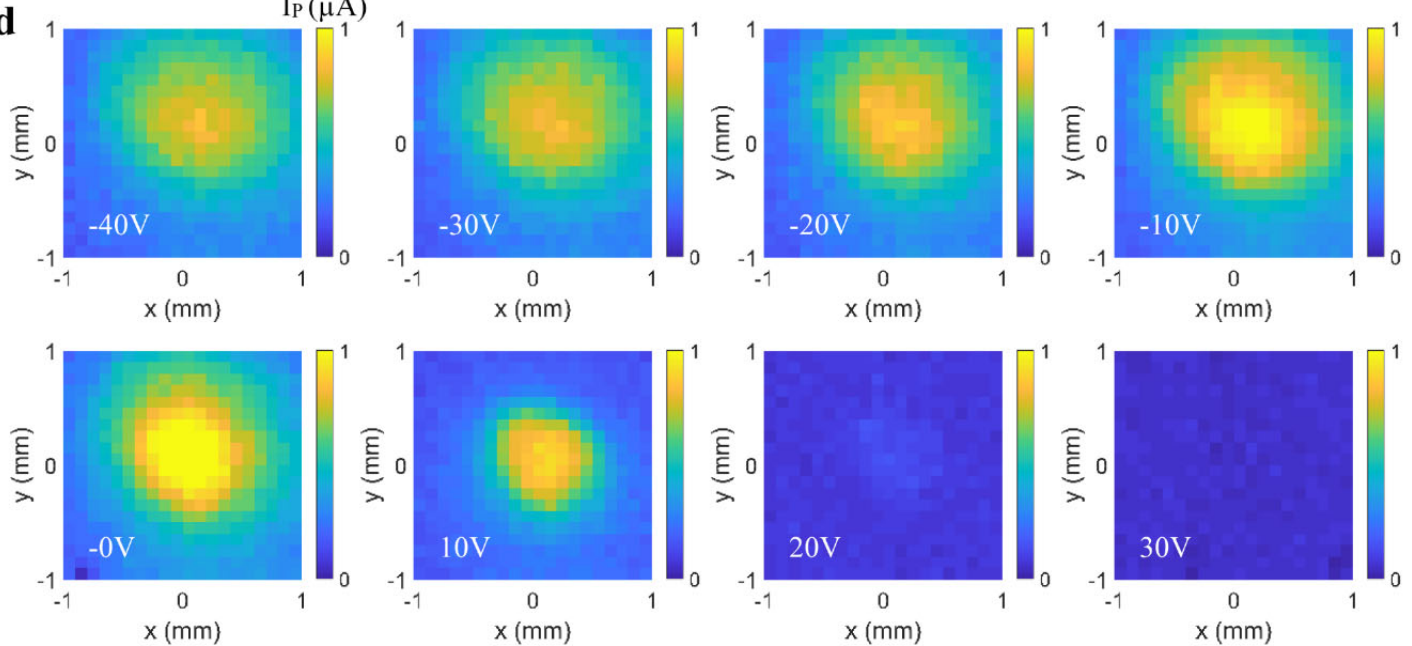

Figure 3. (a-c) Photoresponse to MWIR illumination chopped at frequencies of $210 \mathrm{~Hz}$ (a), $410 \mathrm{~Hz}$ (b) and $700 \mathrm{~Hz}$ (c), with the source-drain voltage of $2.952 \mathrm{~V}$ (a), $2.951 \mathrm{~V}$ (b) and $2.943 \mathrm{~V}$ (c). Gate voltage $\mathrm{V}_{\mathrm{G}}=0 \mathrm{~V}$ for all measurements. $\mathbf{d}$ Photocurrent maps of an IR beam measured under different gate voltages ranging from $-40 \mathrm{~V}$ to $30 \mathrm{~V}$, in steps of $10 \mathrm{~V}$. Source-drain bias voltage is kept at 0.92 V. All measurements are performed at room temperature on another device (Dev\#2).

The device response to modulated IR illumination is also tested by chopping the beam at different frequencies. For details on our characterization method, see Note S4 and Note S5, Supporting Information. Figure 3a-c demonstrate the modulation of the photocurrent at chopping frequencies of $210 \mathrm{~Hz}$ (Figure 3a), $410 \mathrm{~Hz}$ (Figure 3b), and $700 \mathrm{~Hz}$ (Figure 3c). It should be noted that the gradual rise and fall of the photocurrent are caused by the relatively large spot size of the beam that is being 
chopped. Hence, the results of Figure 3a-c do not represent the intrinsic response time of our detector. This is consistent with the fact that the rise/fall times become shorter as the chopping frequency increases. For a simple application, we use our detector to map the intensity profile of the IR beam. Figure $3 \mathrm{~d}$ shows the mapping results under different gate voltages of the detector, ranging from -40 $\mathrm{V}$ to $30 \mathrm{~V}$. The source-drain bias is kept at $\mathrm{V}_{\mathrm{DS}}=0.92 \mathrm{~V}$ for all mapping experiments. It can be seen that the mapped beam photocurrent strongly depends on the applied gate voltage, which is consistent the gate dependence of the detector photoresponse discussed above. The intensity profile with the greatest photocurrent is obtained for gate voltage $\mathrm{V}_{\mathrm{G}}=0 \mathrm{~V}$. Further increase or decrease of the gate voltage leads to a weaker intensity profile due to the reduced responsivity of the detector. In particular, the beam intensity profile is barely observable when the detector is biased at $\mathrm{V}_{\mathrm{G}}=20 \mathrm{~V}$ and $30 \mathrm{~V}$, as for these voltages, the detector is in "silent state" as the graphene channel is n-type doped. The full width half maximum (FWHM) beam diameter is estimated to be around $1.4 \mathrm{~mm}$, based on the profile obtained under $\mathrm{V}_{\mathrm{G}}=0 \mathrm{~V}$.
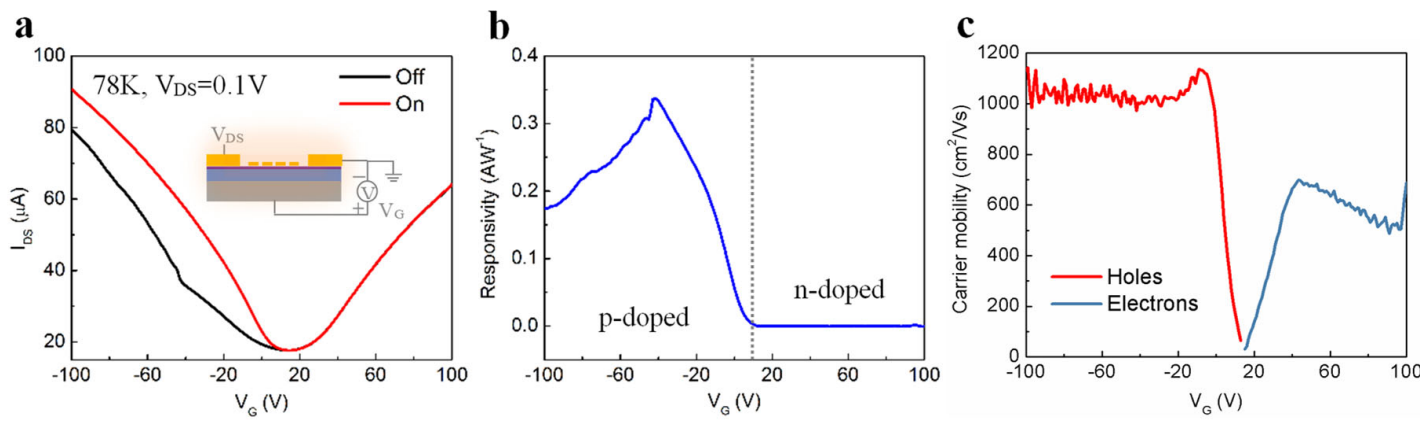

Figure 4. Device characterization of graphene photodetector with integrated JC antennas (Dev\#1) at $78 \mathrm{~K}$. a Transfer characteristic measured under dark and light conditions (globar source, illuminating power $64.6 \mu \mathrm{W}$ ) with gate voltage $\mathrm{V}_{\mathrm{G}}$ swept from $-100 \mathrm{~V}$ to $100 \mathrm{~V}$, and source-drain bias voltage $\mathrm{V}_{\mathrm{DS}}=0.1 \mathrm{~V}$. Inset: Schematic of the detector under test. b Gate-dependent responsivity calculated from photocurrent and illumination power. Photocurrent is obtained by taking difference between source-drain current with and without IR illumination from (a). c Extracted electron (light blue) and hole (dark red) mobilities as a function of gate voltage based on measured electrical properties.

We then measure the device photoresponse at $78 \mathrm{~K}$ and low pressure by mounting the device on a cryostat. Figure 4 a shows the transfer characteristic curves measured under dark and light conditions $\left(\mathrm{V}_{\mathrm{DS}}=0.1 \mathrm{~V}\right)$. At low temperature, the dark current is strongly reduced due to the suppression of thermal generation. Due to the improved carrier mobility at low temperature and reduced Pauli 
blocking, the measured responsivity (Figure 4b) is substantially larger than that measured at room temperature, with a peak value of $0.34 \mathrm{AW}^{-1}$ (corresponds to $424.8 \mathrm{VW}^{-1}$ ) at $\mathrm{V}_{\mathrm{G}}=-42 \mathrm{~V}$. Similar to what we observed at room temperature, the detector shows a gate-switchable photoresponse, featuring a large photoresponse in the p-type doped regime of graphene and a photoresponse close to zero in the n-type doped regime of graphene. The extracted hole mobility (Figure 4c, red curve) shows a similar trend as the responsivity curve under different gate voltages and is larger than the electron mobility (Figure 4c, blue curve). Furthermore, both holes and electrons show increased mobility compared to that at room temperature, probably due to the suppression of lattice scattering from the substrate. ${ }^{[35]}$ The fact that the gate voltage for maximum responsivity $\left(V_{G}=-42 \mathrm{~V}\right)$ is different to that for maximum hole mobility $\left(\mathrm{V}_{\mathrm{G}}=-7 \mathrm{~V}\right)$ might be due to the larger mismatch between the predicted and actual gate-induced carrier density at $78 \mathrm{~K}$, since there is a larger number of vacant trapping centers to be filled by the gate-induced carriers.
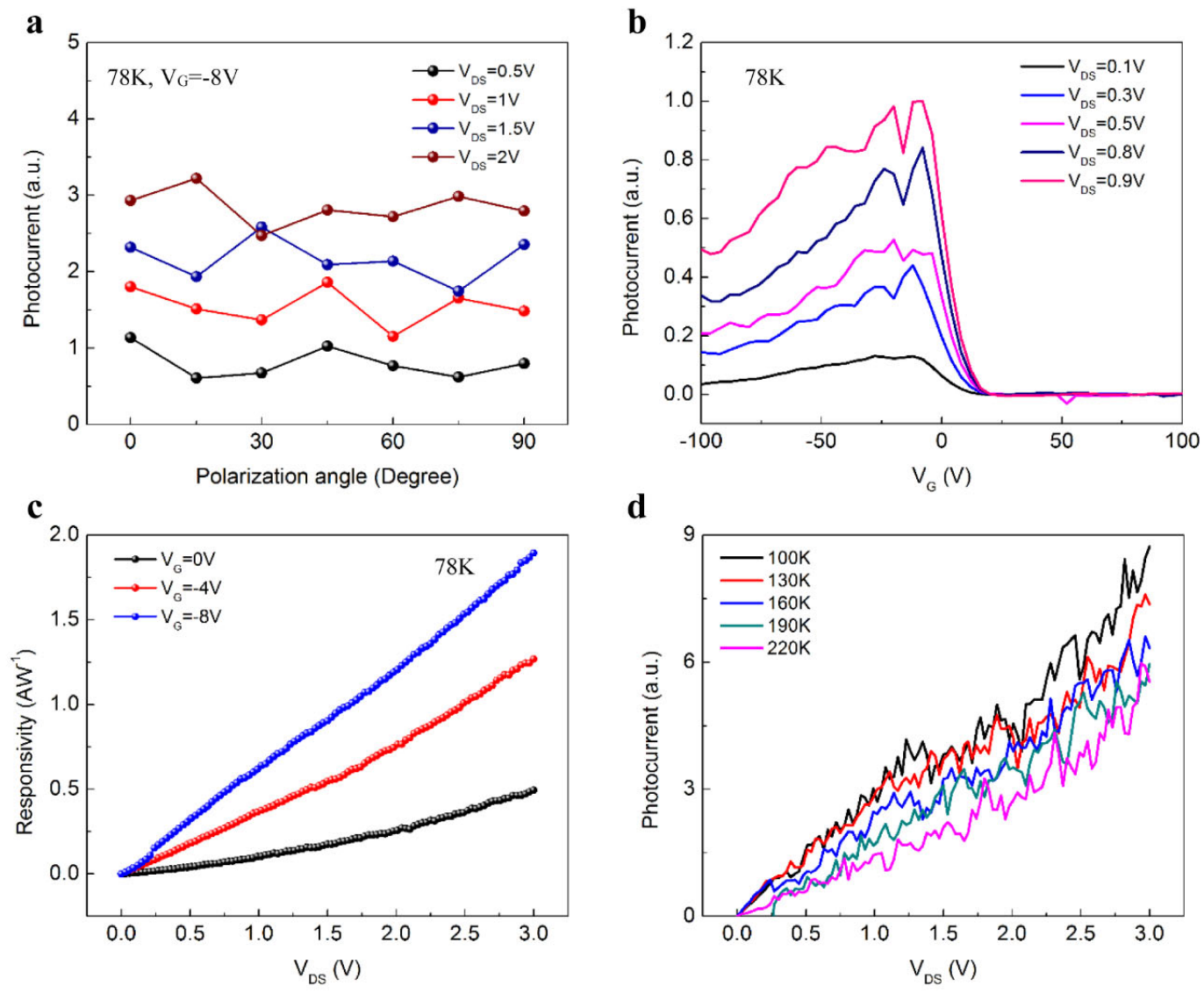
Figure 5. Effects of polarization, source-drain bias, and temperature on photoresponse of graphene detector. a Photocurrent measured at $78 \mathrm{~K}$ as a function of polarization of incident light (globar source) under different source-drain bias voltages, with fixed gate voltage $\mathrm{V}_{\mathrm{G}}=-8 \mathrm{~V}$. b Gate-dependent photocurrent measured at $78 \mathrm{~K}$ under different source-drain bias from $0.1 \mathrm{~V}$ to $0.9 \mathrm{~V}$. c Photoresponsivity as a function of source-drain bias voltage measured at $78 \mathrm{~K}$ under different gate voltages $\left(\mathrm{V}_{\mathrm{G}}=0 \mathrm{~V},-4 \mathrm{~V},-8 \mathrm{~V}\right)$. d Photocurrent measured as a function of source-drain bias voltage from $0 \mathrm{~V}$ to $3 \mathrm{~V}$ at different temperatures $(100 \mathrm{~K}, 130 \mathrm{~K}, 160 \mathrm{~K}, 190 \mathrm{~K}, 220 \mathrm{~K})$, with gate voltage $\mathrm{V}_{\mathrm{G}}$ $=-8 \mathrm{~V}$. Note that measurements in (a, c-d) performed on another device (Dev\#3) on the same chip as Dev\#1.

We further characterize the polarization dependence of our graphene detector. Figure 5a shows the photocurrent measured as the incident polarization is varied from $0^{\circ}$ to $90^{\circ}$ (with respect to the $\mathrm{x}$-axis) under different source-drain bias voltages. Due to the polarization-independent absorption enhancement in graphene that arises from the symmetric shape of the JC antennas, the detector exhibits a largely polarization-insensitive photoresponse. The effect of source-drain bias on the detector photoresponse is also investigated in Figure 5b-c. Figure 5b shows the gate-dependent photocurrents under different source-drain bias voltages, demonstrating a consistent increase of photocurrent as source-drain bias voltage increases from $0.1 \mathrm{~V}$ to $0.9 \mathrm{~V}$. This is due to the increased carrier drift velocity and collection efficiency as a result of the increased electric field between source and drain electrodes. The linear relationship between the source-drain bias and the detector responsivity is evident in Figure 5c, where the responsivity is measured at $\mathrm{V}_{\mathrm{DS}}$ swept from $0 \mathrm{~V}$ to 3 $\mathrm{V}$ for $\mathrm{V}_{\mathrm{G}}=0 \mathrm{~V},-4 \mathrm{~V}$ and $-8 \mathrm{~V}$. Notably, a responsivity of $1.9 \mathrm{AW}^{-1}$ (which corresponds to $\sim 4.4 \times 10^{3}$ $\mathrm{VW}^{-1}$ ) is obtained with $\mathrm{V}_{\mathrm{G}}=-8 \mathrm{~V}$ and $\mathrm{V}_{\mathrm{DS}}=3 \mathrm{~V}$. The photocurrent is also characterized at different temperatures (Figure 5d). Due to the stronger carrier scattering at higher temperatures, the photocurrent decreases as the temperature increases from $100 \mathrm{~K}$ to $220 \mathrm{~K} .{ }^{[34]}$

Finally, we characterize the detector photoresponse in the visible and near-infrared. Figure 6a shows the measured photocurrent at $\mathrm{V}_{\mathrm{DS}}=3 \mathrm{~V}$ with monochromatic illumination $(\lambda=532 \mathrm{~nm}, 650 \mathrm{~nm}$, and $850 \mathrm{~nm}$ ) from a laser-driven light source filtered by a monochromator. The gate bias is set to be $V_{G}$ $=0 \mathrm{~V}$ so that the graphene is p-type doped and detector is in its "working state". The light is chopped at a frequency of $1 \mathrm{kHz}$. The current output from the detector is amplified with a current preamplifier, whose output is monitored with an oscilloscope. It can be seen that our detector exhibits a strong and 
broadband photoresponse across the visible and near-infrared regime, despite the fact that the antennas are designed to enhance the graphene absorption in the MWIR region. The simulated graphene absorption is less than 3\% in the visible and near-infrared (See in Note S13, Supporting Information). The responsivities are calculated to be $\sim 28 \mathrm{AW}^{-1}, 74 \mathrm{AW}^{-1}$ and $118 \mathrm{AW}^{-1}$ at wavelengths of $532 \mathrm{~nm}, 650 \mathrm{~nm}$ and $850 \mathrm{~nm}$, respectively. We attribute the relatively high responsivity in the visible and near-infrared region compared to related previous reports ${ }^{[22,24 a]}$ to the increased carrier collection efficiency by our JC antennas. Figure $6 \mathrm{~b}$ shows the device temporal responses measured with illumination $(\lambda=532 \mathrm{~nm}, 650 \mathrm{~nm}$, and $850 \mathrm{~nm})$ from a pulsed supercontinuum laser filtered by a tunable filter. From these results, we find that the rise times $\left(\tau_{1}\right.$, defined as the $10 \%$ to $90 \%$ intervals) are $\tau_{1} \approx 3 \mathrm{~ns}$ for the three wavelengths. The fall time is estimated to be $\tau_{2} \approx 0.746 \mu$ s at $\lambda=532 \mathrm{~nm}$. This value is obtained by fitting the exponential decay region of the curve. The fitting results for all wavelengths are provided in Note S14, Supporting Information. Our detector thus shows fast temporal response, with rise time of a few nanoseconds and submicrosecond fall times, which are much shorter than previous works that employ carrier trapping centers $(\sim 100 \mathrm{~s})^{[25]}$ or photogating effects $(\sim 1-3 \mathrm{~ms})^{[26-27]}$.
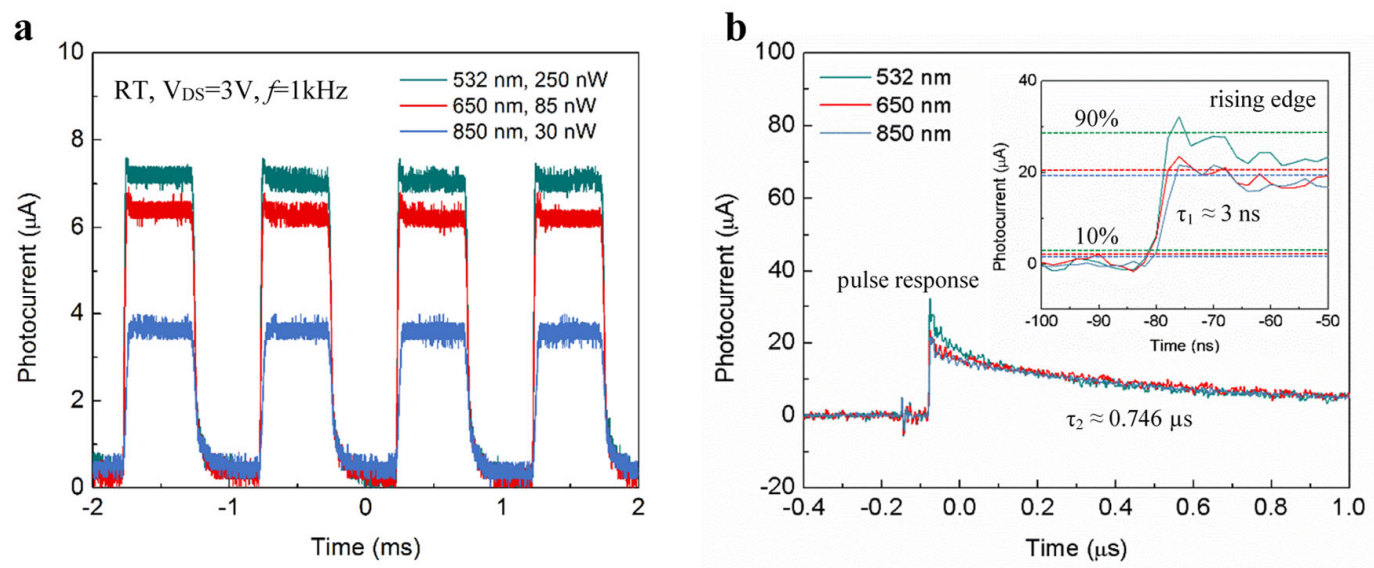

Figure 6. Photoresponse in the visible and near-infrared spectral region (Dev\#2). a Measured photocurrent in response to chopped monochromatic illumination $(\lambda=532 \mathrm{~nm}, 650 \mathrm{~nm}$, and $850 \mathrm{~nm}$, chopping frequency $\mathrm{f}=1 \mathrm{kHz}$ ) from a laser-driven light source filtered by a monochromator. Device is biased with $\mathrm{V}_{\mathrm{DS}}=3 \mathrm{~V}, \mathrm{~V}_{\mathrm{G}}=0 \mathrm{~V}$. b Temporal response measured with illumination $(\lambda=532 \mathrm{~nm}$, $650 \mathrm{~nm}$, and $850 \mathrm{~nm}$ ) from a pulsed laser (output pulse width: $2 \mathrm{~ns}$ ) filtered by a tunable filter. Device is biased with $\mathrm{V}_{\mathrm{DS}}=3 \mathrm{~V}, \mathrm{~V}_{\mathrm{G}}=0 \mathrm{~V}$. All measurements are performed at room temperature.

\section{Conclusions}


In conclusion, we demonstrate a polarization-independent graphene photoconductor with integrated Jerusalem-cross nanoantennas operating in the mid-wave infrared. Photoresponse is measured at both room and cryogenic temperatures. Compared to previously reported work employing end-to-end rod antennas, the unique shape of the JC-antennas, with their elongated ends, and the narrower gaps ( 40 $\mathrm{nm}$ ) between adjacent antennas, render a stronger and polarization-independent optical absorption enhancement in graphene. Our photocurrent measurements confirm the largely polarizationinsensitive photoresponse of the detector. Unlike previously demonstrated detectors based on trapping centers or photogating effects which show response times of milliseconds or even hundreds of seconds, our detector shows a fast response with a measured rise time of 3 ns. Notably, our graphene detector also exhibits a strong and broadband photoresponse at visible and near-infrared wavelengths, which we attribute to the improved carrier collection by the antennas. We believe our work provides guidelines to the design of antenna-assisted graphene photodetectors with improved responsivities and versatile optical properties.

\section{Supporting Information}

Supporting Information is available from the Wiley Online Library or from the author.

\section{Acknowledgements}

M.Y. and Y.G. contributed equally to this work. M.Y. and Y.G. performed the simulations and fabricated the devices. M.Y. conducted the measurements, with contributions from S.B., Y.G. and J.C.. M.Y. analysed the data, with contributions from Y.G., S.B. and K.B.C.. M.Y., Y.G. and K.B.C. wrote the manuscript, with contributions from all authors. All authors have given approval for the final version of the manuscript.

This work was supported by DARPA (HR0011-16-1-004), and by the Australian Research Council (DP180104141). This work was performed in part at Melbourne Centre for Nanofabrication (MCN) in Victorian Node of Australian National Fabrication Facility (ANFF).

\section{Conflict of Interest}

The authors declare no conflict of interest.

\section{References}

[1] J. L. Miller, Principles of infrared technology, Springer, 1994. 
[2] a) C. Young, S.-S. Kim, Y. Luzinova, M. Weida, D. Arnone, E. Takeuchi, T. Day, B. Mizaikoff, Sensors and Actuators B: Chemical 2009, 140, 24; b) D. Rodrigo, O. Limaj, D. Janner, D. Etezadi, F. J. G. De Abajo, V. Pruneri, H. Altug, Science 2015, 349, 165.

[3] T. Day, M. Pushkarsky, D. Caffey, K. Cecchetti, R. Arp, A. Whitmore, M. Henson, E. B. Takeuchi, presented at Technologies for Optical Countermeasures X; and High-Power Lasers 2013: Technology and Systems 2013.

[4] a) F. K. Tittel, D. Richter, A. Fried, in Solid-state mid-infrared laser sources, Springer 2003, p. 458; b) K. Zhang, L. Zhang, F. L. Yap, P. Song, C. W. Qiu, K. P. Loh, Small 2016, 12, 1302.

[5] a) B. Guo, Y. Wang, C. Peng, H. Zhang, G. Luo, H. Le, C. Gmachl, D. Sivco, M. Peabody, A. Y. Cho, Optics express 2004, 12, 208; b) M. Razeghi, B.-M. Nguyen, Reports on Progress in Physics 2014, 77, 082401; c) X. Tang, M. M. Ackerman, M. Chen, P. Guyot-Sionnest, Nature Photonics 2019, 13, 277.

[6] a) H. Figgemeier, C. Ames, J. Beetz, R. Breiter, D. Eich, S. Hanna, K.-M. Mahlein, T. Schallenberg, A. Sieck, J. Wenisch, presented at Infrared Technology and Applications XLIV 2018; b) C. Cervera, N. Baier, O. Gravrand, L. Mollard, C. Lobre, G. Destefanis, J. Zanatta, O. Boulade, V. Moreau, presented at Infrared Technology and Applications XLI 2015.

[7] E. N. Lewis, I. W. Levin, Applied spectroscopy 1995, 49, 672.

[8] B. Weng, J. Qiu, L. Zhao, Z. Yuan, C. Chang, Z. Shi, presented at Quantum Sensing and Nanophotonic Devices XI 2014.

[9] G. Sarusi, Infrared physics \& technology 2003, 44, 439.

[10] a) A. Haddadi, A. Dehzangi, R. Chevallier, S. Adhikary, M. Razeghi, Scientific reports 2017, 7, 3379; b) A. Haddadi, R. Chevallier, G. Chen, A. Hoang, M. Razeghi, Applied Physics Letters 2015, 106, 011104; c) A. M. Hoang, A. Dehzangi, S. Adhikary, M. Razeghi, Scientific reports 2016, 6, 24144.

[11] a) G. Konstantatos, M. Badioli, L. Gaudreau, J. Osmond, M. Bernechea, F. P. G. De Arquer, F. Gatti, F. H. Koppens, Nature nanotechnology 2012, 7, 363; b) I. Nikitskiy, S. Goossens, D. Kufer, T. Lasanta, G. Navickaite, F. H. Koppens, G. Konstantatos, Nature communications 2016, 7, 11954; c) S. A. McDonald, G. Konstantatos, S. Zhang, P. W. Cyr, E. J. Klem, L. Levina, E. H. Sargent, Nature materials 2005, 4, 138.

[12] a) S. Keuleyan, E. Lhuillier, V. Brajuskovic, P. Guyot-Sionnest, Nature Photonics 2011, 5, 489; b) X. C. Yu, Y. Y. Li, X. N. Hu, D. L. Zhangg, Y. Tao, Z. X. Liu, Y. M. He, M. A. Haque, Z. Liu, T. Wu, Q. J. Wang, Nature Communications 2018, 9; c) M. M. Ackerman, X. Tang, P. Guyot-Sionnest, ACS Nano 2018, 12, 7264; d) Z. Deng, K. S. Jeong, P. Guyot-Sionnest, ACS Nano 2014, 8, 11707.

[13] F. Niklaus, C. Vieider, H. Jakobsen, presented at MEMS/MOEMS technologies and applications III 2008.

[14] J. Piotrowski, Opto-Electron. Rev 2004, 12, 111.

[15] a) Y. Yao, R. Shankar, P. Rauter, Y. Song, J. Kong, M. Loncar, F. Capasso, Nano letters 2014, 14, 3749; b) S. Du, W. Lu, A. Ali, P. Zhao, K. Shehzad, H. Guo, L. Ma, X. Liu, X. Pi, P. Wang, Advanced Materials 2017, 29, 1700463.

[16] X. Yu, P. Yu, D. Wu, B. Singh, Q. Zeng, H. Lin, W. Zhou, J. Lin, K. Suenaga, Z. Liu, Nature communications 2018, 9, 1.

[17] Q. Liang, Q. Wang, Q. Zhang, J. Wei, S. X. Lim, R. Zhu, J. Hu, W. Wei, C. Lee, C. Sow, Advanced Materials 2019, 31, 1807609.

[18] Q. Guo, A. Pospischil, M. Bhuiyan, H. Jiang, H. Tian, D. Farmer, B. Deng, C. Li, S.-J. Han, H. Wang, Nano letters 2016, 16, 4648.

[19] a) S. Bae, H. Kim, Y. Lee, X. Xu, J.-S. Park, Y. Zheng, J. Balakrishnan, T. Lei, H. R. Kim, Y. I. Song, Nature nanotechnology 2010, 5, 574; b) B. Deng, Z. Pang, S. Chen, X. Li, C. Meng, J. Li, M. Liu, J. Wu, Y. Qi, W. Dang, ACS nano 2017, 11, 12337.

[20] a) F. Koppens, T. Mueller, P. Avouris, A. Ferrari, M. Vitiello, M. Polini, Nature nanotechnology 2014, 9, 780; b) R. R. Nair, P. Blake, A. N. Grigorenko, K. S. Novoselov, T. J. Booth, T. Stauber, N. M. Peres, A. K. Geim, Science 2008, 320, 1308; c) K. F. Mak, L. Ju, F. Wang, T. F. Heinz, Solid State Communications 2012, 152, 1341.

[21] a) A. De Sanctis, G. F. Jones, D. J. Wehenkel, F. Bezares, F. H. Koppens, M. F. Craciun, S. Russo, Science advances 2017, 3, e1602617; b) F. Xia, T. Mueller, Y.-m. Lin, A. Valdes-Garcia, P. Avouris, Nature nanotechnology 2009, 4, 839; c) M. Freitag, T. Low, W. Zhu, H. Yan, F. Xia, P. Avouris, Nature communications 2013, 4, 1951; d) T. Deng, Z. Zhang, Y. Liu, Y. Wang, F. Su, S. Li, Y. Zhang, H. Li, H. 
Chen, Z. Zhao, Nano letters 2019, 19, 1494; e) S. Castilla, B. Terrés, M. Autore, L. Viti, J. Li, A. Y. Nikitin, I. Vangelidis, K. Watanabe, T. Taniguchi, E. Lidorikis, Nano letters 2019, 19, 2765.

[22] T. Mueller, F. Xia, P. Avouris, Nature photonics 2010, 4, 297.

[23] K. F. Mak, M. Y. Sfeir, Y. Wu, C. H. Lui, J. A. Misewich, T. F. Heinz, Physical review letters 2008, 101, 196405.

[24] a) N. M. Gabor, J. C. Song, Q. Ma, N. L. Nair, T. Taychatanapat, K. Watanabe, T. Taniguchi, L. S. Levitov, P. Jarillo-Herrero, Science 2011, 334, 648; b) M. Badioli, A. Woessner, K.-J. Tielrooij, S. Nanot, G. Navickaite, T. Stauber, F. García de Abajo, F. H. Koppens, Nano letters 2014, 14, 6374.

[25] Y. Zhang, T. Liu, B. Meng, X. Li, G. Liang, X. Hu, Q. J. Wang, Nature communications 2013, 4, 1811.

[26] X. Yu, Y. Li, X. Hu, D. Zhang, Y. Tao, Z. Liu, Y. He, M. A. Haque, Z. Liu, T. Wu, Nature communications 2018, 9, 4299.

[27] C.-H. Liu, Y.-C. Chang, T. B. Norris, Z. Zhong, Nature nanotechnology 2014, 9, 273.

[28] A. Safaei, S. Chandra, M. W. Shabbir, M. N. Leuenberger, D. Chanda, Nature communications 2019, 10, 1.

[29] S. Cakmakyapan, P. K. Lu, A. Navabi, M. Jarrahi, Light: Science \& Applications 2018, 7, 20.

[30] F. M. Monavar, N. Komjani, Progress In Electromagnetics Research 2011, 121, 103.

[31] Q. Shao, G. Liu, D. Teweldebrhan, A. A. Balandin, 2008, 92, 202108.

[32] M. Iqbal, A. K. Singh, M. Iqbal, J. Eom, Journal of Physics: Condensed Matter 2012, 24, 335301.

[33] a) E. J. Lee, K. Balasubramanian, R. T. Weitz, M. Burghard, K. Kern, Nature nanotechnology 2008, 3, 486; b) E. C. Peters, E. J. Lee, M. Burghard, K. Kern, Applied Physics Letters 2010, 97, 193102.

[34] W. Zhu, V. Perebeinos, M. Freitag, P. Avouris, Physical Review B 2009, 80, 235402.

[35] S.-i. Takagi, A. Toriumi, M. Iwase, H. Tango, IEEE Transactions on Electron Devices 1994, $41,2357$. 


\section{University Library}

\section{- M M I N E R VA \\ A gateway to Melbourne's research publications}

Minerva Access is the Institutional Repository of The University of Melbourne

Author/s:

Ye, M;Gao, Y;Cadusch, JJ;Balendhran, S;Crozier, KB

Title:

Mid-Wave Infrared Polarization-Independent Graphene Photoconductor with Integrated Plasmonic Nanoantennas Operating at Room Temperature

Date:

2021-01-20

\section{Citation:}

Ye, M., Gao, Y., Cadusch, J. J., Balendhran, S. \& Crozier, K. B. (2021). Mid-Wave Infrared Polarization-Independent Graphene Photoconductor with Integrated Plasmonic Nanoantennas Operating at Room Temperature. ADVANCED OPTICAL MATERIALS, 9 (6), https://doi.org/10.1002/adom.202001854.

Persistent Link:

http://hdl.handle.net/11343/294858 